\title{
Subarachnoid block with ultrasound guided transversalis fascia plane block for cesarean section: a randomized controlled double-blind study
}

\author{
ME Aydin ${ }^{1}$, Z Bedir ${ }^{2}$, AM Yayik ${ }^{3}$, EC Celik ${ }^{3}$, I Ates ${ }^{1}$, E Oral Ahiskalioglu and A Ahiskalioglu
}

1. Ataturk University School of Medicine, Anesthesiology and Reanimation, Erzurum, Turkey

2. Nenehatun State Hospital, Anesthesiology and Reanimation, Erzurum, Turkey

3. Regional Training Research Hospital, Anesthesiology and Reanimation, Erzurum, Turkey

Background and aims The transversalis fascia plane block originally defined by Hebbard in 2009 was performed for postoperative analgesia in T12-L1 dermatomes (1). The TFP block has provided effective analgesia in various surgeries such as inguinal hernia repair, iliac graft surgery, cesarean section. As far as we know, this is the first study of the postoperative analgesic efficacy of TFP block in cesarean section under spinal anesthesia. We share the preliminary results of our ongoing study here.

Materials and methods The study was planned to include 60 patients in Group TFP and Group Control. At the end of surgery, USG guided, $20 \mathrm{ml} 0.25 \%$ bupivacaine to Group 1 and $20 \mathrm{ml}$ of SF to Group 2 were injected into bilateral Transversalis Fascia Plane (figure 1). Postoperative analgesia was maintained with morphine PCA and intermittent paracetamol infusions. Rescue analgesic was determined as $50 \mathrm{mcg}$ IV fentanyl.

Results Pain scores were lower in the TFP group at all time points, this difference was not statistically significant. Morphine consumption was significantly lower in Group TFP than Group C at the all time periods $(\mathrm{p}<0.05)$ (figure 2).

Conclusions After cesarean section under spinal anesthesia, TFP block was found to be effective in postoperative analgesia control. In cesarean, transversus abdominis plane block and quadratus lumborum block have been used as a complement to multimodal analgesia in postoperative analgesia control. But the TFP block is a more applicable block compared to TAP and QLB. Because the risk of peritoneal penetration is lower than the TAP block and its applicability in the supine position is easier than QLB. Consequently, in cesarean section, the TFP block achieved effective analgesia by reducing postoperative opioid consumption and VAS scores in comparison to the control group.
Figure 1

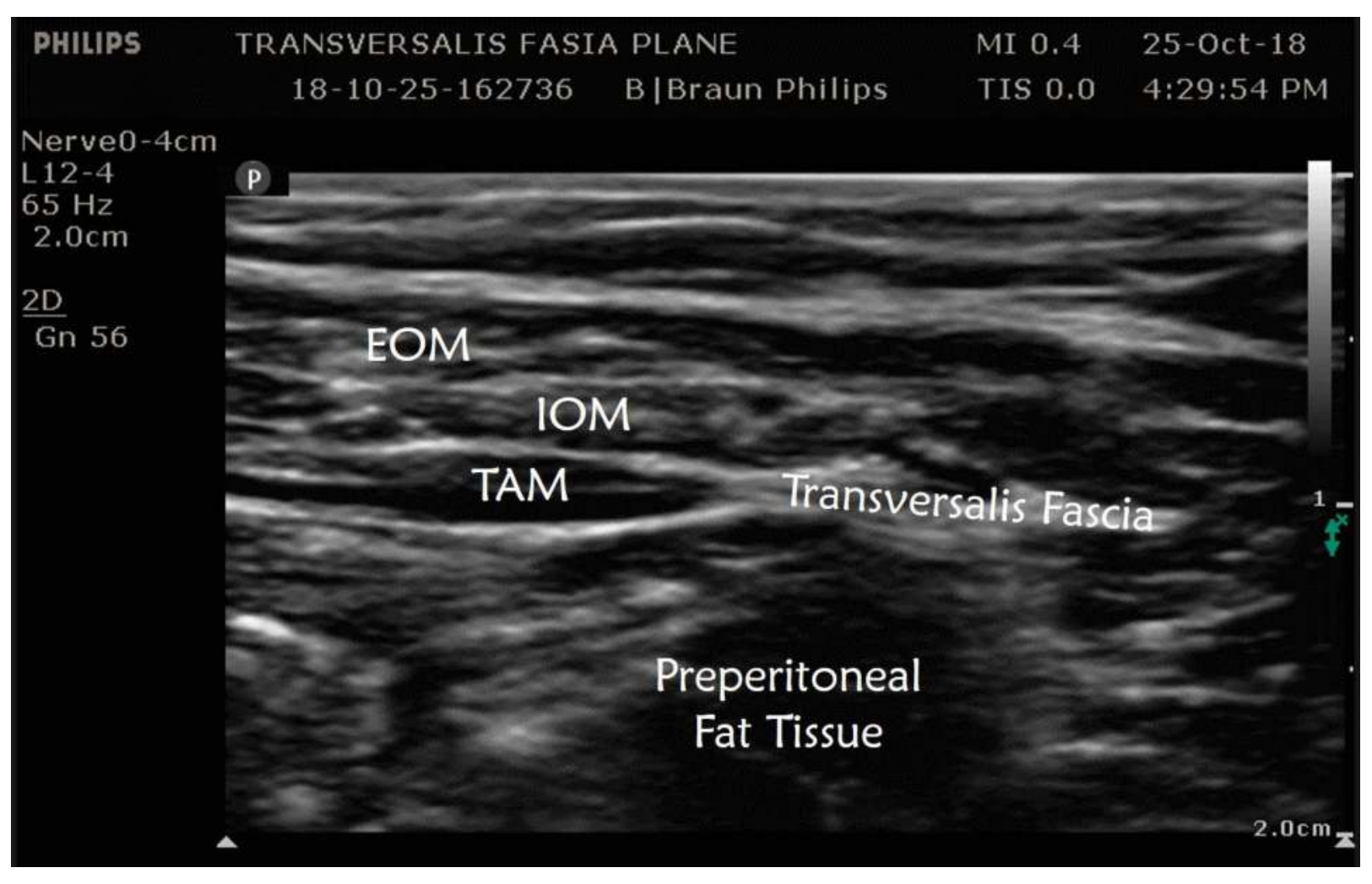

Figure 2

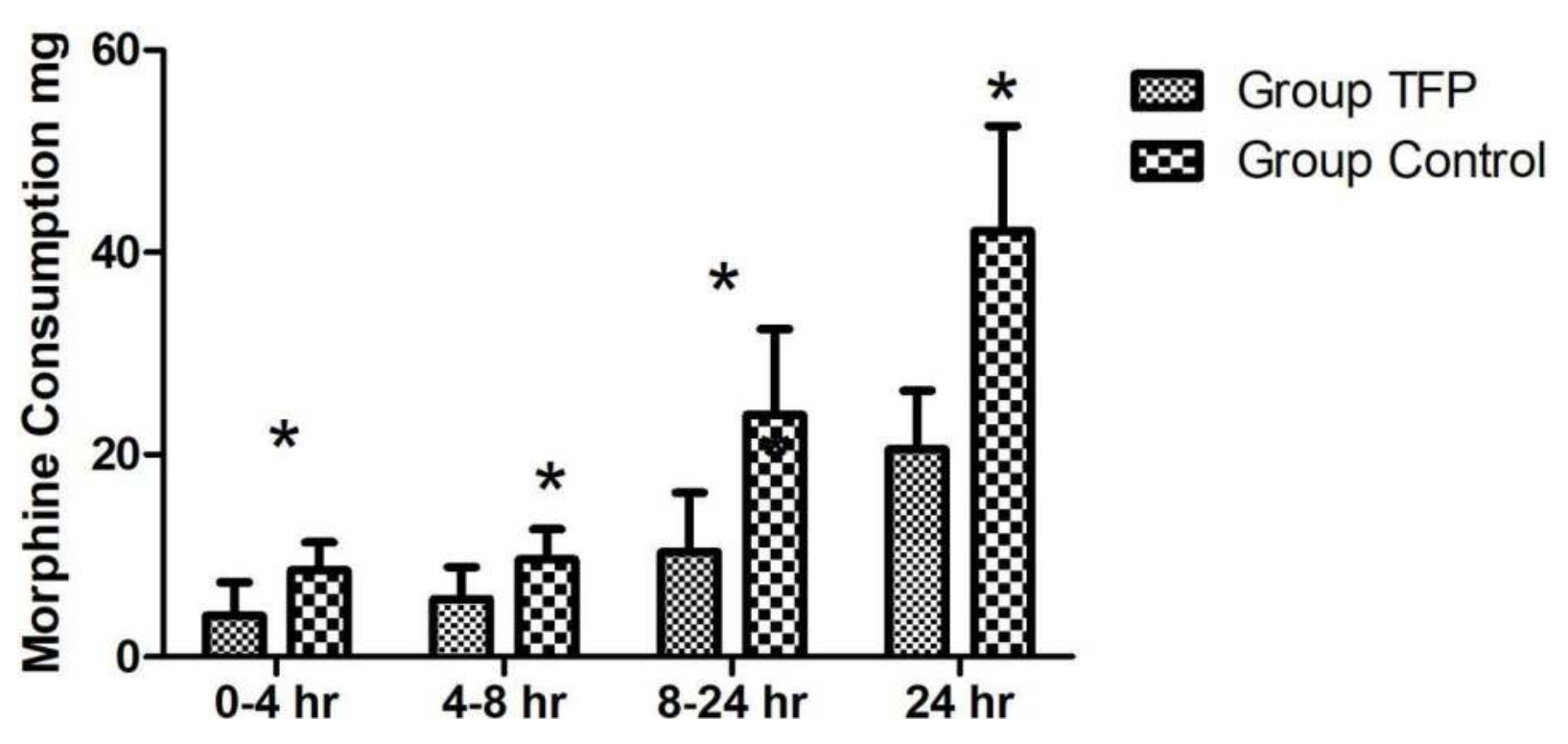

